\title{
Synthetic circular multi-miR sponge simultaneously inhibits miR-21 and miR-93 in esophageal carcinoma
}

\author{
Zhe Wang ${ }^{1,2} \cdot \mathrm{Ke} \mathrm{Ma}^{2} \cdot$ Yulan Cheng ${ }^{2} \cdot$ John M. Abraham ${ }^{2} \cdot \mathrm{Xi} \mathrm{Liu}^{3} \cdot \mathrm{Xiquan}_{\mathrm{Ke}}^{4} \cdot$ Zhirong Wang $^{1} \cdot$ \\ Stephen J. Meltzer ${ }^{2}$
}

Received: 11 January 2019 / Revised: 29 April 2019 / Accepted: 13 May 2019 / Published online: 19 June 2019

(c) The Author(s), under exclusive licence to United States and Canadian Academy of Pathology 2019

\begin{abstract}
MicroRNAs (miRs) are post-transcriptional regulators involved in the initiation and progression of many tumors. Recently, naturally occurring circular RNAs (circRNAs) have been described in eukaryotic cells:; they comprise a new class of gene regulators. Naturally occurring circular miR sponges, which induce miR loss-of-function, can prevent endogenous oncomiRs from binding to their cognate mRNA targets. These findings suggest that synthetic (artificial) circular RNAs could be constructed as therapeutic molecular sponges to suppress harmful onco-miRs. Using enzymatic ligation, we designed and constructed a circular RNA containing both miR-21 and miR-93 binding sites. The synthetic circular sponge was resistant to digestion with RNase R. Luciferase assays and functional experiments showed that the circular multi-miR sponge was more stable than its linear counterpart. Moreover, endogenous miR-21 and miR-93 were inhibited by the circular sponge. In addition, the synthetic sponge significantly suppressed cellular proliferation and migration while promoting apoptosis in esophageal carcinoma cells. Finally, in a murine xenograft model, the circular sponge significantly inhibited tumor growth in vivo. Taken together, these findings establish that the design and construction of efficient artificial miR sponges represent a novel strategy to achieve miR loss-of-function in molecular cancer therapeutics.
\end{abstract}

\section{Introduction}

MicroRNAs (miRs) comprise a class of small non-coding RNAs that modulate translational repression or degradation of messenger RNA (mRNA) molecules by binding to 3' untranslated regions (UTRs) of target mRNAs containing

These authors contributed equally: Zhe Wang, Ke Ma

Stephen J. Meltzer

smeltzer@jhmi.edu

1 Department of Gastroenterology, Tongji Hospital, Tongji University School of Medicine, Shanghai 200092, China

2 Division of Gastroenterology, Department of Medicine, Sidney Kimmel Comprehensive Cancer Center, Johns Hopkins University School of Medicine, Baltimore, MD 21205, USA

3 Department of Pathology, The First Affiliated Hospital of Xi' an Jiaotong University, No. 277 Yanta West Road, Xi' an 710061 Shaanxi, China

4 Department of Gastroenterology, The First Affiliated Hospital of Bengbu Medical College, Bengbu 233004 Anhui, China
miR response elements (MREs) [1]. MiRs have been extensively implicated in critical biological processes including proliferation, apoptosis, and differentiation of cells and tissues; moreover, aberrant miR expression is closely linked to the initiation and progression of numerous cancers [2]. Therefore, methods of inhibiting miRs represent a logical strategy for future molecular anti-tumor therapeutics.

Currently, artificially induced miR loss-of-function strategies fall into two main categories: 1) chemically modified antisense oligonucleotides or 2) $\mathrm{miR}$ sponges [3, 4]. MiR sponges, which have been described heretofore only as native (naturally occurring) molecules, contain at least one miR binding site to regulate target mRNA expression by sequestering cognate miRs [4]. Native miR sponges include pseudogene RNAs, long non-coding RNAs, viral RNAs, and other types. Recently, circular RNAs (circRNAs) have received attention as a type of naturally occurring, endogenous miR sponge [5]. CircRNAs are covalently closed circular structures lacking 5'-3' polarity or a polyadenylated tail and are resistant to exoribonucleasemediated degradation, making them more stable than their linear counterparts [6, 7]. Accumulating evidence 


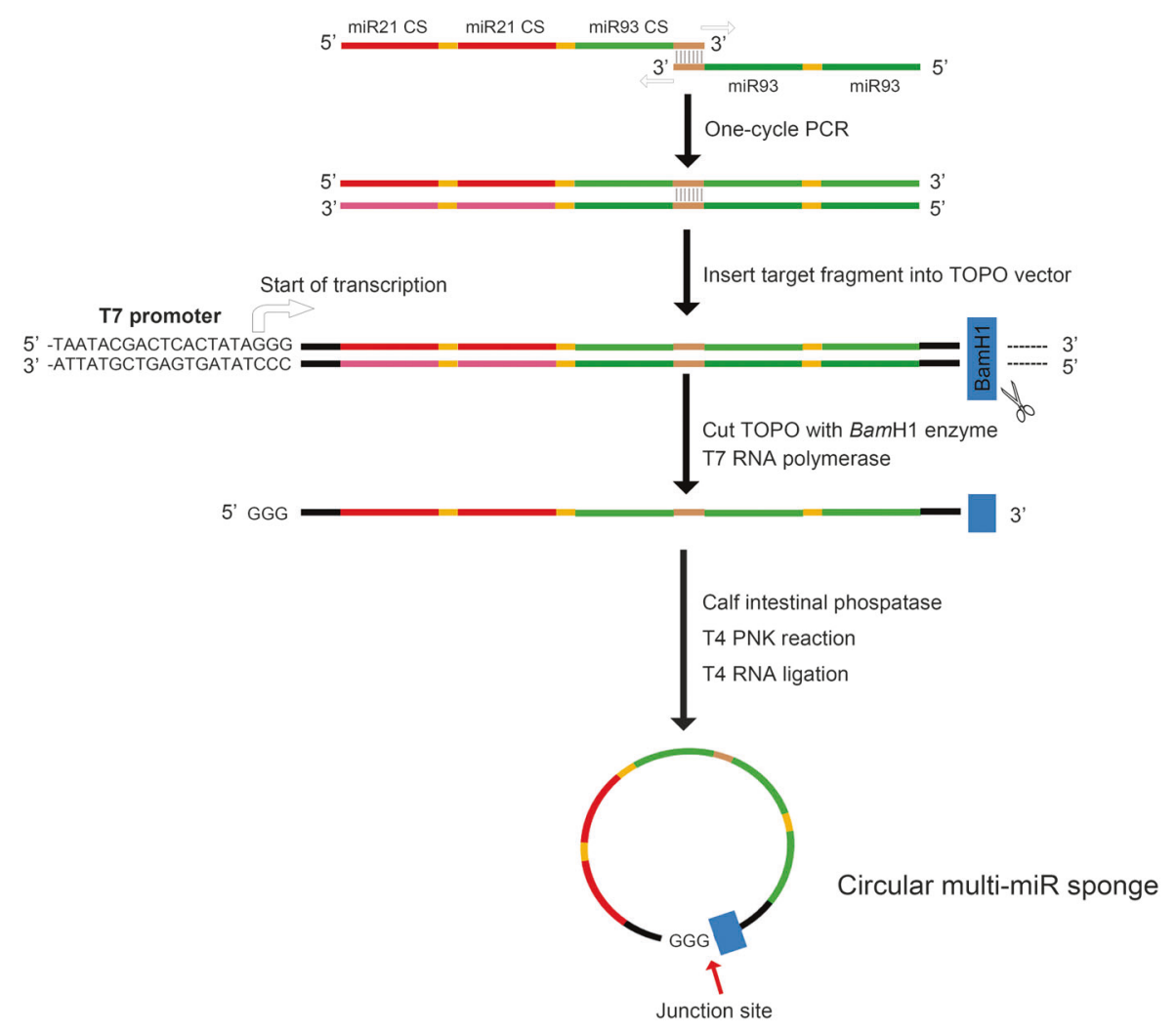

Fig. 1 Workflow for producing a synthetic circular multi-miR sponge against miR-21 and miR-93. First, two DNA oligonucleotides were designed. Sense strand contains two repeated miR-21 complementary sequence (CS) and one miR-93 CS, respectively. Antisense strand contains two miR-93 complete sequence. Yellow lines stand for a short spacer with 4-nucleotide residues ("CCAA"). Brown lines represent nucleotide region space to help to hybridize to one another after heat denaturation. After one-cycle PCR reaction, generated double-stranded DNA containing two miR-21 binding site and three miR-93 binding site was cloned into pCR 2.1-TOPO TA vector (Invitrogen). Then, BamH1 enzyme was used to cut TOPO vector and T7 RNA polymerase was utilized to produce abundant linear RNAs with multi-miR binding sites. The start of transcription site is GGG of T7 promoter. Next, Calf intestinal phosphatase dephosphorylates the 5' terminal of the single linear RNA transcript, and T4 polynucleotide kinase generates a 5' phosphorylation suitable for ligation. Incubation with T4 RNA ligase 1 results in RNA circularization. Red arrow indicates junction site of circular multi-miR sponge demonstrates that due to selectively conserved miR binding sites, a large proportion of circRNAs can function as native miR sponges, sequestering miRs and thereby relieving the repression of their downstream cognate target mRNAs [5, 8]. Thus far, synthetic miR sponges have consisted exclusively of linear RNA transcripts containing multiple tandem miR-complementary sequences transcribed from a strong promoter in viral or non-viral gene vectors $[9,10]$. Recent explorations of native circRNAs suggest that artificially engineered synthetic miR sponges comprise a promising new molecular strategy for achieving RNA interference therapy.

Esophageal carcinoma (EC) is one of the most common human malignant tumors, being seventh in incidence and sixth in cancer-related mortality worldwide [11]. Like many other tumors, EC is a multifactorial disease, involving diverse environmental and genetic factors [12, 13]. In the last decade, several miRs have been found to be dysregulated in EC and to participate in EC initiation and progression [14]. MiR-21, one of most well-characterized such miRs, is highly expressed in most cancers including EC [15] and considered an oncomiR, since it is associated with cellular proliferation, apoptosis, migration and progression by targeting key tumor suppressor genes, including programmed cell death 4 (PDCD4) [16] and phosphatase and tensin homolog (PTEN) [17]. We previously reported that another oncomiR, miR-93, is overexpressed in EC cells and associated with increased cell proliferation, cycle progression and in vivo tumorigenesis [18]. Therefore, we chose miR-21 and miR-93 as synthetic circRNA targets in a novel molecular therapeutic strategy against EC.

In this study, we designed and constructed a circular RNA simultaneously containing both miR-21 and miR-93 binding sites. Competitive inhibition of miR-21 and miR-93 activity showed that the synthetic circular RNA functioned as a miR sponge, significantly sequestering endogenous miR-21 and miR-93. Moreover, functional assays demonstrated that the synthetic circular multi-miR sponge 
inhibited proliferation and migration while promoting apoptosis in EC cells. In conclusion, we have developed a novel molecular strategy to inhibit oncogenic miRs based on the construction of a synthetic circRNA as a miR sponge.

\section{Materials and Methods}

\section{Design of circular sponge}

First, two sense and antisense DNA oligonucleotides containing miR-21 and miR-93 binding sites were designed to produce linear RNA molecules prior to synthesis of circular RNA. The DNA oligonucleotides were designed to contain 2 repeated miR-21 binding sites and 3 repeated miR-93 binding sites perfectly complementary to miR-21 and miR93, respectively. To establish enough dimensionality for miR-21 and miR-93 binding, adjacent miR binding sites were separated by a spacer using 4-nucleotide residues ("CCAA"). In addition, each oligonucleotide contained a 21-nucleotide region at its respective 3' end that allowed it to hybridize to the other oligonucleotide after heat denaturation. The sequences of the two oligonucleotides were 5'-ATCAACATCAGTCTGATAAGCTACCAATCAAC ATCAGTCTGATAAGCTACCAACTACCTGCACGAAC AGCACTTTGTAGATAAATGAAGA-3' (sense) and 5'ACAAAGTGCTGTTCGTGCAGGTAGCCAACAAAGT GCTGTTCGTGCAGGTAGTCTTCATTTATCTACAAA GTG-3' (antisense). Next, the designed DNA oligonucleotides were ordered (IDT, Inc., city state) for subsequent synthesis. As a control, we designed scrambled of miR binding sites. The sequence of the scrambled miR site was 5'-ACTTAGTGTCTATGCGTATGT-3'.

\section{Synthesis of circular sponge}

Synthesis is illustrated schematically in Fig. 1. First, a one-cycle PCR with parameters of 95 degrees x 30 s, 65 degrees x $30 \mathrm{~s}$, and 72 degrees x 1 minute was performed using a $25 \mathrm{ng}$ of the designed sense or antisense DNA oligonucleotide to generate double-stranded DNA, which was then cloned into pCR 2.1-TOPO TA vector (Invitrogen, Carlsbad CA). After Sanger sequencing, TOPO vector containing the correctly orientated sequence was chosen and digested with BamHI enzyme (New England Biolabs, Inc., Ipswich, MA) and purified by QIAprep ${ }^{\circledast}$ Spin Miniprep Kit (Qiagen, Hilden, Germany). Subsequently, we used T7 RNA polymerase according to HiScribe ${ }^{\mathrm{TM}}$ T7 High Yield RNA Synthesis Kits' instructions (New England Biolabs, Inc., Ipswich, MA) to produce abundant linear RNAs containing multiple miR binding sites, which were then cleaned up by RNeasy ${ }^{\oplus}$ MinElute ${ }^{\oplus}$ Cleanup Kits (Qiagen). Next, we needed to circularize this single-stranded linear RNA. The linear RNA was first treated with calf intestinal alkaline phosphatase (New England Biolabs) to nonspecifically catalyze the dephosphorylation of 5 , and 3' of RNA phosphomonoesters, followed by incubation with T4 polynucleotide kinase (New England Biolabs) to provide 5' phosphorylation for the subsequent ligation reaction. Finally, the linear RNA was circularized with T4 RNA ligase 1 (New England Biolabs). After each step of the cyclization process, purification of products was performed. Synthetic circular sponge concentration was measured by ND-1000 V3.3.0 software; the sponge was then stored at $-80{ }^{\circ} \mathrm{C}$ for subsequent experiments. Scrambled circular RNA was synthesized similarly.

\section{Reverse transcription-polymerase chain reaction (RT-PCR) and Sanger sequencing}

To verify the closed-loop structure of the synthetic circular multi-miR sponge, reverse-transcribed cDNA was amplified by TopTaq Master Mix kit (Qiagen) using divergent or convergent primers. Next, Sanger sequencing was performed on PCR products generated with divergent primers to confirm the end-to-end ligation junction regions. Primers were as follows: divergent, 5'-AGCACACTGGCGGC CGTTAC-3' (sense) and 5'-ATATCCATCACACTGGC GG-3' (antisense), Convergent, 5'-AATTGGGCCCTCTA GATGCA-3' (sense) and 5'-ACTAGTAACGGCCGCCA GTG-3' (antisense).

\section{RNase R treatment}

The circular multi-miR sponge and its linear counterpart were treated with 2U/ug RNase R (Epicentre Biotechnologies) or non-enzyme control buffer at $37^{\circ} \mathrm{C}$ for 30 minutes. After purification with QIAprep Spin Miniprep kits (Qiagen), RNA samples were analyzed by $1.0 \%$ TAE-agarose gel electrophoresis.

\section{Cell culture}

Human EC cell lines (SKGT4, OE33, and FLO-1) were obtained from Sigma-Aldrich. Normal esophageal epithelial cells (HEEPiC) was obtained from ScienCell Research Laboratories. The cell lines were identified by PCRamplified short tandem repeat analysis. Human EC cell line JHEso-Ad1 was a gift from Johns Hopkins University School of Medicine. EC cell lines were cultured in DMEM medium (Gibco) supplemented with $10 \%$ fetal bovine serum (FBS, Gibco) and 1\% penicillin-streptomycin (Gibco). HEEPiC was cultured in EpiCM-2 medium 
(\#4121, ScienCell). All cells were incubated at $37^{\circ} \mathrm{C}$ with $5 \% \mathrm{CO}_{2}$.

\section{Transfection of circular sponges}

SKGT4 and OE33 cell lines were seeded at a density of $2.0 \times 10^{5} /$ well in 6-well plates prior to transfection. Then, circular multi-miR sponge (circ-21-93) or scrambled circular RNA (circ-scr) at a concentration of $5 \mathrm{nM}$ were transfected into cells using lipofectamine RNAiMax (Invitrogen) according the manufacturer's instructions. After incubation for $24 \mathrm{~h}$, cells were collected for subsequent functional experiments.

\section{Quantitative real-time PCR}

qRT-PCR was utilized to measure expression levels of miR21 and miR-93. Total RNA from EC cell lines SKGT4, OE33, JHEso-Ad1, FLO-1 and normal esophageal epithelial cells (HEEPiC) were extracted using TRIzol reagent (Ambion) and reverse-transcribed. Subsequently, cDNA samples were amplified using TaqMan MicroRNA Assays (Applied Biosystems, Foster City, CA) on an ABI 7900HT Fast Real-Time PCR system. Expression levels were defined based on the threshold cycle $(\mathrm{Ct})$ and calculated using the $2^{-\Delta \Delta C t}$ formula. RNU6B was used as a reference gene.

To measure mRNA expression levels, total RNAs from SKGT4 and OE33 cells transfected with circ-21-93 or circ-scr were extracted as described above. After reverse transcription, cDNA was generated and amplified using SYBR PrimeScript ${ }^{\mathrm{TM}}$ (Bio-Rad, Hercules, CA). Ct value was normalized to GAPDH expression. Primer sequences were: PTEN, 5'-ACTTGAAGGCGTATACAGGAACA$3^{\prime}$ (sense) and 5'-CACCAGTTCGTCCCTTTCCA-3' (antisense); PDCD4, 5'-GTCCCTTTGGAAGTCTTC TACC-3' (sense) and 5'-TTCTACAAACCGCTCCAG CA-3' (antisense); TGF $\beta$ R2, 5'-GGAAGATGCTGCTT CTCCAAAG-3' (sense) and 5'-GAGGCTGATGCC TGTCACTT-3' (antisense); GAPDH, 5'-GGTATCGTG GAAGGACTCATGAC-3' (sense) and 5'-ATGCCAGT GAGCTTCCCGTTCAG-3' (antisense).

\section{Stability assays}

To evaluate the stability of this circular multi-miR sponge, we transfected the circular sponge (circ-21-93) or its counterpart linear RNA (lin-21-93) into SKGT4 cells. Cells were harvested at various time points $(1,6,12,24 \mathrm{~h})$ after transfection and subjected to qRT-PCR to measure circ- or lin-21-93 expression. Levels of circ-21-93 or lin-21-93 at 6, 12, $24 \mathrm{~h}$ post-transfection were normalized to their respective expression levels at $1 \mathrm{~h}$. GAPDH was used as a reference gene.

\section{Dual-Glo luciferase assays}

First, we successfully constructed pmirGLO DualLuciferase vector (Promega, Madison, WI) containing miR-21 or miR-93 target sequences by inserting either miR21 or miR-93 binding sites using PmeI and XbaI restriction enzymes (New England Biolabs). Next, $1.0 \times 10^{4}$ cells/well of SKGT4 and OE33 were seeded in 96-well plates and cotransfected with different concentrations $(2,3,5 \mathrm{nM})$ of either circ-21-93 or circ-scr and $80 \mathrm{ng}$ pmirGLO miR-21/ miR-93 target site vector. $24 \mathrm{~h}$ after transfection, cells were analyzed for Firefly luciferase activity using the Dual-Glo ${ }^{\circ}$ Luciferase Assay Kit (Promega) and VICTOR2 fluorometry (Perkin Elmer, Waltham, MA) and normalized to Renilla luciferase activity.

\section{Cell proliferation assays}

Cells were harvested $24 \mathrm{~h}$ after transfection and then reseeded into 96-well plates at a final density of $1.0 \times 10^{3}$ /well. At incubation times of $0,1,3$, and 5 days, 10 ul of WST-1 reagent (Roche) was added to each well and cells were incubated for an additional $2 \mathrm{~h}$, after which optical density (OD) values of treated cells were detected on a microplate reader (Molecular Devices, Sunnyvale, CA, USA).

\section{Cell colony assays}

For colony assays, $1.0 \times 10^{3}$ cells per well were seeded in 6well plates. After 10 days of incubation, the plates were washed twice with PBS and dyed with Diff-Quik Fixative, Diff-Quik Solution I and Diff-Quik Solution II (Dade Behring Inc, Newark, DE) for 10 minutes successively at room temperature. The numbers of colonies formed were assessed using Image $\mathbf{J}$ software.

\section{Cell scratch assays}

Transfected cells were incubated for $24 \mathrm{~h}$ to grow into a monolayer. A $200 \mu \mathrm{l}$ filter tip was used to draw a straight line to create a wound area, then incubation was continued for $48 \mathrm{~h}$. The extent of wound healing was observed and photographed under a microscope at $0,24,48 \mathrm{~h}$. Finally, scratch healing rate was analyzed using Image $\mathbf{J}$ software.

\section{Cell apoptosis assays}

FITC Annexin V/ Dead Cell Apoptosis Kits (Invitrogen) were used to measure apoptosis. After incubation for $24 \mathrm{~h}$, transfected cells were collected and washed twice with cold PBS. Then, $100 \mu \mathrm{L}$ of $1 \times$ annexin-binding buffer was added to resuspend cells, followed by the addition of $5 \mu \mathrm{L}$ 

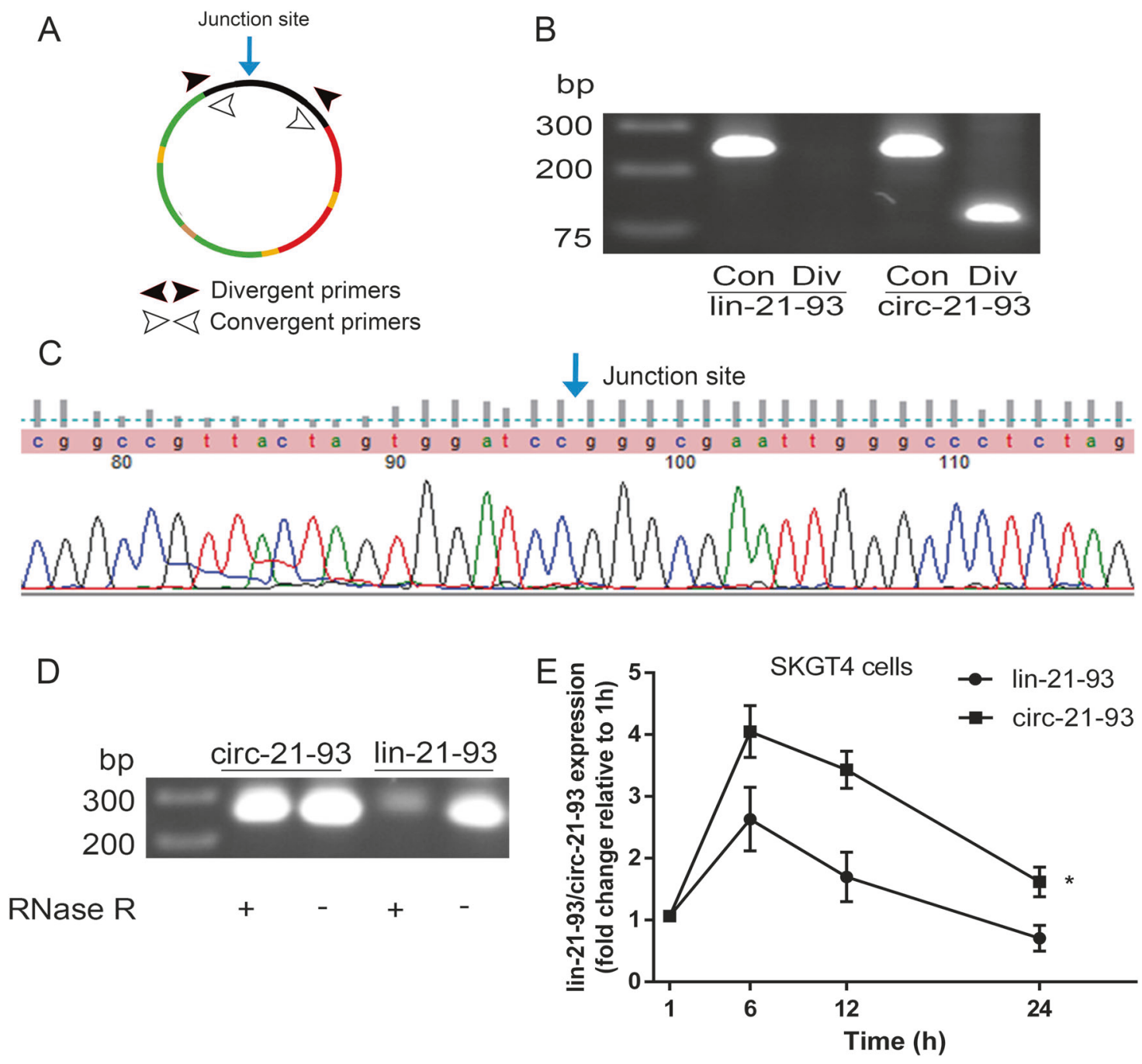

Fig. 2 Verification of artificial circular multi-miR sponge. A Schematic diagram of annealing sites of convergent and divergent primer sets for the artificial circular multi-miR sponge. Red lines represent repeated miR-21 complementary sequences. Green lines represent repeated miR-93 complementary sequences. Blue arrows indicate head-to-tail junctions. B Results of RT-PCR of linear multi-miR sponge (lin-21-93) and circular multi-miR sponge (circ-21-93) using convergent ('Con') or divergent primers ('Div'). PCR products amplified from divergent primers contained the end-to-end ligation

of FITC annexin V and I $\mu$ l of $100 \mu \mathrm{g} / \mathrm{mL}$ PI working solution. Subsequently, following $15 \mathrm{~min}$ incubation, flow cytometry was used to analyze the stained cells after adding $400 \mu \mathrm{L}$ of $1 \times$ annexin-binding buffer.

\section{Immunofluorescence staining}

Cells were seeded in Chamber Slide (Nunc, Inc., Naperville, IL) and transfected with either circ-scr or circ-21-93. After $24 \mathrm{~h}$, cells were fixed in acetone and blocked with 5\% normal goat serum at room temperature for $1 \mathrm{~h}$. Then cells were stained with primary antibodies and secondary antibodies in junction regions. C The head-to-tail junction of circ-21-93 was confirmed by Sanger sequencing of divergent RT-PCR products. Blue arrows indicate end-to-end ligation junction regions. D Stabilities of lin-21-93 and circ-21-93 were assessed by RNase R. Circ-21-93 was more resistant to RNase R digestion than its linear counterpart, lin-2193. E qRT-PCR was performed to further evaluate stabilities of circ21-93 and lin-21-93 at various digestion time points. Fold changes of circ-21-93 and lin-21-93 were normalized to their respective $\mathrm{Ct}$ values at 1 h. $* p<0.05$

sequence. Primary antibodies used were rabbit anti cleaved caspase-3 (1:400; \#9661, Cell Signaling Technology). Secondary antibodies were AlexaFluor 568 goat anti-rabbit IgG (1:500; A-11036, Invitrogen). Next, nuclei were counterstained with Hoechst 33342 (Thermo-Fisher Scientific).

\section{Murine xenograft model}

Four 5- to 6-week-old athymic female nude mice were purchased from Charles River (Wilmington, MA), under a protocol approved by the Johns Hopkins University Animal Care and Use Committee (Protocol Number: M019M07). $2.0 \times 10^{6}$ 
A

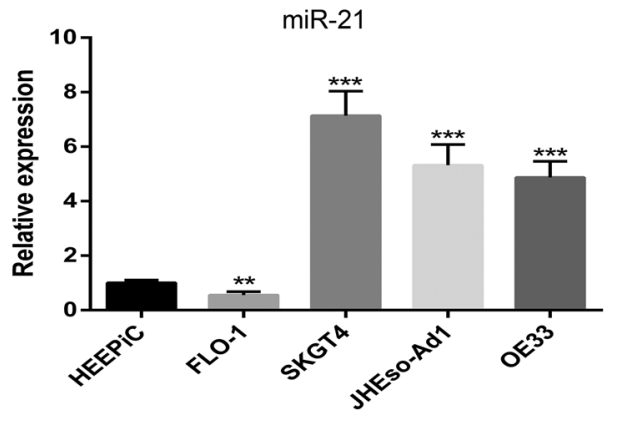

B

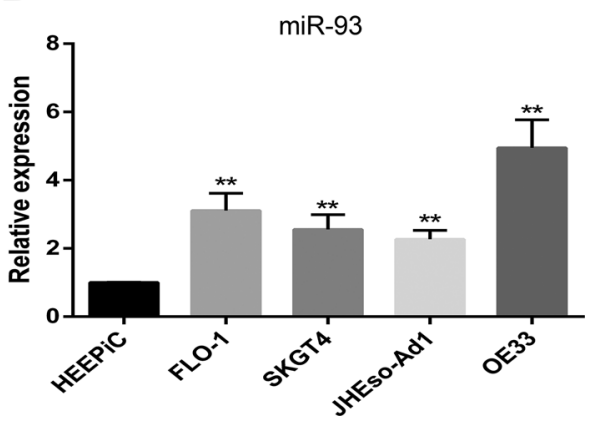

Fig. 3 Expression levels of miR-21 and miR-93 in EC cells. A Expression levels of miR-21 in esophagus cancer cells vs. the immortalized normal esophagus cell line, HEEPiC. B Expression levels of miR-93 in esophagus cancer cells $v s$. HEEPiC. $* * p<0.01 ; * * * p<0.001$

SKGT4 cells transfected with either circ-scr or circ-21-93 were resuspended in $200 \mu$ l of injection solution (PBS: Matrigel $=1: 1)$ and injected subcutaneously into dorsal flanks of nude mice. Each mouse carried two explants, one with circ21-93 and one with circ-scr treatment. Tumor growth was monitored after one week by measuring tumor sizes three times in one week. Length $(L)$ and width $(W)$ of each mouse were measured with calipers, and tumor volumes were calculated using the formula $V=\left(W^{2} \times L\right) / 2$. Four weeks after injection, mice were euthanized.

\section{Statistical analyses}

Means \pm SEM were displayed as representative values for data in figures. Discrepancies among group variables were analyzed by Student's unpaired/paired t-test. SPSS 20.0 was used for all statistical analyses and $p<0.05$ was defined as a statistical significance cutoff. All experiments were repeated at least three times.

\section{Results}

\section{Construction and verification of artificial circular multi-miR sponge}

Figure 1 depicts the biosynthesis of the circular multi-miR sponge. After synthesis, RT-PCR and Sanger sequencing were used to verify correct construction of the sponge. PCR products amplified from divergent primers spanned the endto-end ligation junction regions, while PCR products from convergent primers contained 2 repeated miR-21 binding sites and 3 repeated miR-93 binding sites (Fig. 2A). RTPCR results showed that both the circular multi-miR sponge (circ-21-93) and its linear counterpart (lin-21-93) yielded expected products when using convergent primers; however, divergent primers generated the expected products only from circ-21-93 (Fig. 2B). Moreover, Sanger sequencing of the divergent primer product confirmed the correct end-to-end ligation junction region sequence of circ21-93 (Fig. 2C). In addition, circ-21-93 was treated with RNase R to test its stability, with lin-21-93 serving as a control for. Results showed that circ-21-93 was resistant to digestion by RNase R vs. lin-21-93 (Fig. 2D). Next, we transfected circ-21-93 or lin-21-93 into SKGT4 cells to further evaluate the stability of circ-21-93. Results revealed that relative to lin-21-93, circ-21-93 levels in SKGT4 cells were more abundant at various time points posttransfection. Moreover, at $6 \mathrm{~h}$, circ-21-93 levels escalated more quickly than did lin-21-93 levels, while degradation of circ-21-93 during the next $6 \mathrm{~h}$ was slower compared to lin-21-93 (Fig. 2E). Taken together, these findings establish that circ-21-93 was successfully and accurately synthesized as well as more stable than its linear RNA counterpart.

\section{Expression levels of miR-21 and miR-93 in EC cells}

Native miR-21 and miR-93 expression levels were first measured by qRT-PCR to define the best natural model system for functional assays in EC and normal esophageal cells. Relative to normal esophageal cell HEEPiC, levels of miR-21 were significantly higher in SKGT4, JHEso-Ad1 and OE33 EC cell lines, but lower in FLO-1 cancer cells. Levels of miR-21 were highest in SKGT4 cells, 7-fold levels in HEEPiC (Fig. 3A). Levels of miR-93 were higher in all 4 EC cell lines than that in HEEPiC cells, with the highest level seen in OE33, more than four-fold higher than in HEEPiC (Fig. 3B). For this reason, SKGT4 and OE33 EC cell lines were chosen for subsequent experiments.

\section{Biologic effects of synthetic circular multi-miR sponge in vitro}

To examine biologic effects of the circular multi-miR sponge against miR-21 and miR-93, luciferase reporter assays were conducted after co-transfection of of SKGT4 and OE33 cells with either circ-21-93 or circ-scr plus a luciferase reporter construct, pmirGLO, containing either the miR-21 (Luc21) or 
Fig. 4 Effectiveness of synthetic circular multi-miR sponge.

A, B, SKGT4 cells cotransfected with different concentrations $(2,3,5 \mathrm{nM})$ of either circ-scr or circ-21-93 and $80 \mathrm{ng}$ luciferase vector containing miR-21 target site (Luc21) or miR-93 target site (Luc93). C, D In OE33 cells, different concentration $(2,3,5$ $\mathrm{nM}$ ) of circ-scr / circ-21-93 and either Luc21 or Luc93 were cotransfected into cells. Firefly luciferase activity was normalized to that of Renilla luciferase. $* p<0.05 ; * * p<0.01$; $* * * p<0.001$
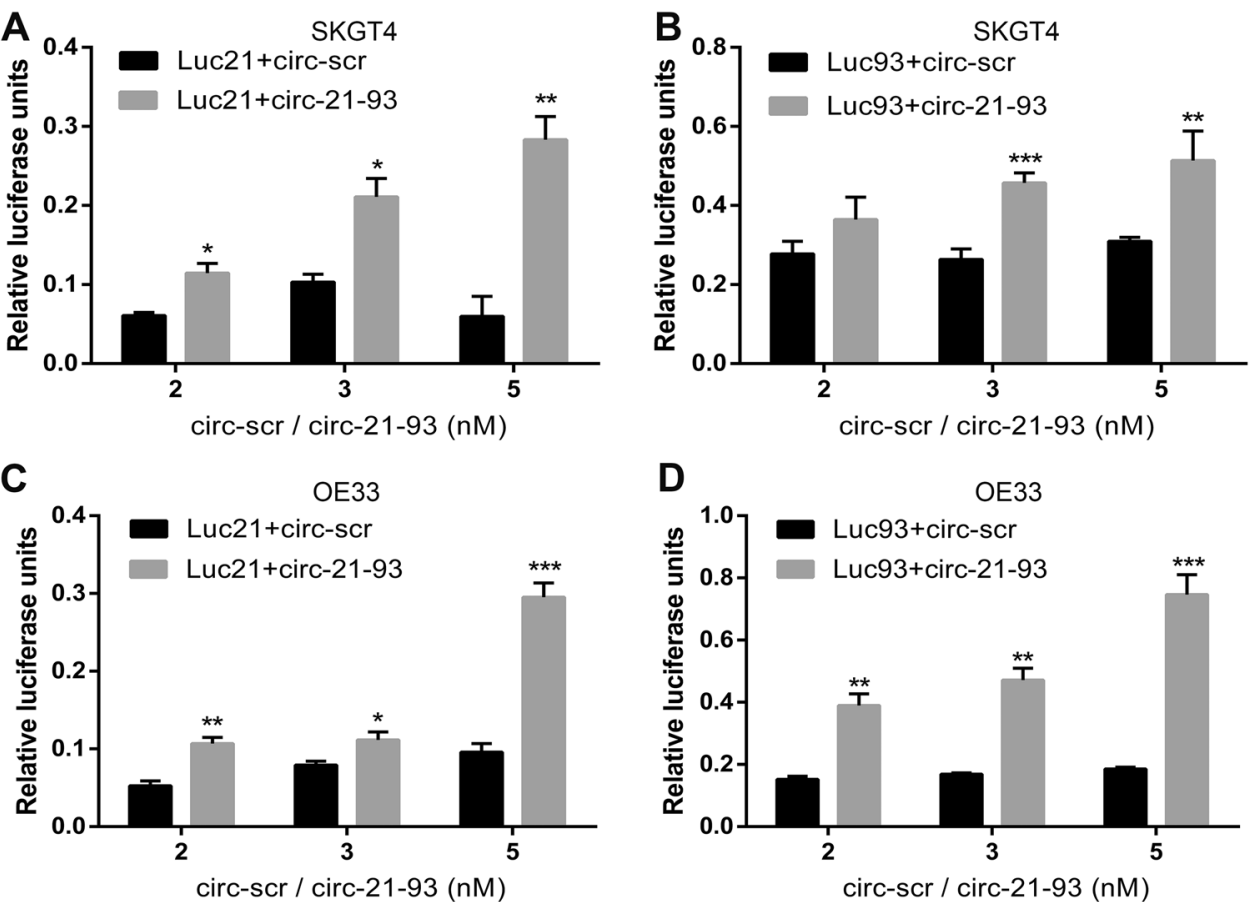

the miR-93 target site (Luc93). If our synthetic circular RNA worked successfully, we expected it to counteract the inhibitory effects of native miR-21 and miR-93 on their target sites, Thus, we predicted that circ-21-93 should de-repress the luciferase reporter vector by competitively binding to and removing miR-21 and miR-93 from the vector.

This was indeed what we found: results are shown in Fig. 4. In SKGT4 cells, luciferase activity of cells transfected with circ-21-93 was significantly higher than in cells transfected with the scrambled control circ-scr, regardless of whether the luciferase reporter vector contained the miR-21 binding site (Luc21, Fig. 4A) or the miR-93 binding site (Luc93, Fig. 4B). Moreover, this effect was also dose-dependent: as the concentration of circ-21-93 increased, this stimulatory effect on luciferase activity became stronger, in contrast to the circ-scr control, with maximal effect at the highest concentration of 5 nM (Fig. 4A, B). Similar effects were observed in OE33 cells, with luciferase increasing by 3-fold and 5-fold for Luc21 and Luc93 at a concentration of $5 \mathrm{nM}$, respectively (Fig. 4C, D). For this reason, the $5 \mathrm{nM}$ concentration of circ-21-93 was used for subsequent functional studies.

\section{The synthetic circular multi-miR sponge inhibits proliferation and migration while promoting apoptosis in EC cells}

First, cellular proliferation after treatment with circ-21-93 was assessed by WST-1 assays. Results showed that cells transfected with circ-21-93 significantly decreased proliferation of SKGT4 cells at days 3 and 5 relative to circ-scr $(p=0.0272$ and 0.0093 , Fig. 5A); similar results were seen in OE33 cells at day 5 ( $p=0.0109$, Fig. 5B). Next, colonyforming capacity was effectively inhibited by circ-21-93 relative to circ-scr in both SKGT4 and OE33 $(p<0.01$ and $p<0.05$, Fig. 5C, D). Moreover, cell migration was also inhibited by circ-21-93. Specifically, scratch assays revealed that treatment with circ-21-93 caused substantial reductions in cell migration at 24 and $48 \mathrm{~h}$ relative to circscr in SKGT4 cells $(p<0.05$ and $p<0.01$, Fig. 5E, F). Finally, we evaluated the effect of the synthetic circular multi-miR sponge on apoptosis quantitively and qualitatively. Flow cytometry showed that SKGT4 cells transfected with circ-21-93 exhibited significantly greater apoptosis than did cells treated with circ-scr $(p<0.05$, Fig. 5G, both panels). Results of immunofluorescence staining showed that levels of cleaved caspase-3 were higher in both SKGT4 and OE33 cells treated with circ-2193 than in cells treated with circ-scr, proving that circ-21-93 induced increased apoptosis (Fig. 6).

\section{Synthetic circular multi-miR sponge alters downstream target mRNA expression}

As onco-miRs, miR-21 and miR-93 mediate at least some of their effects by binding to target tumor suppressor gene transcripts. Previous research has verified three well-known tumor suppressor genes, PTEN, PDCD4 and TGF $\beta R 2$, as major targets of both miR-21 and miR-93 [19-23]. We predicted that messenger RNA levels of these targets, which are downregulated by miR-21 and miR-93, would be 

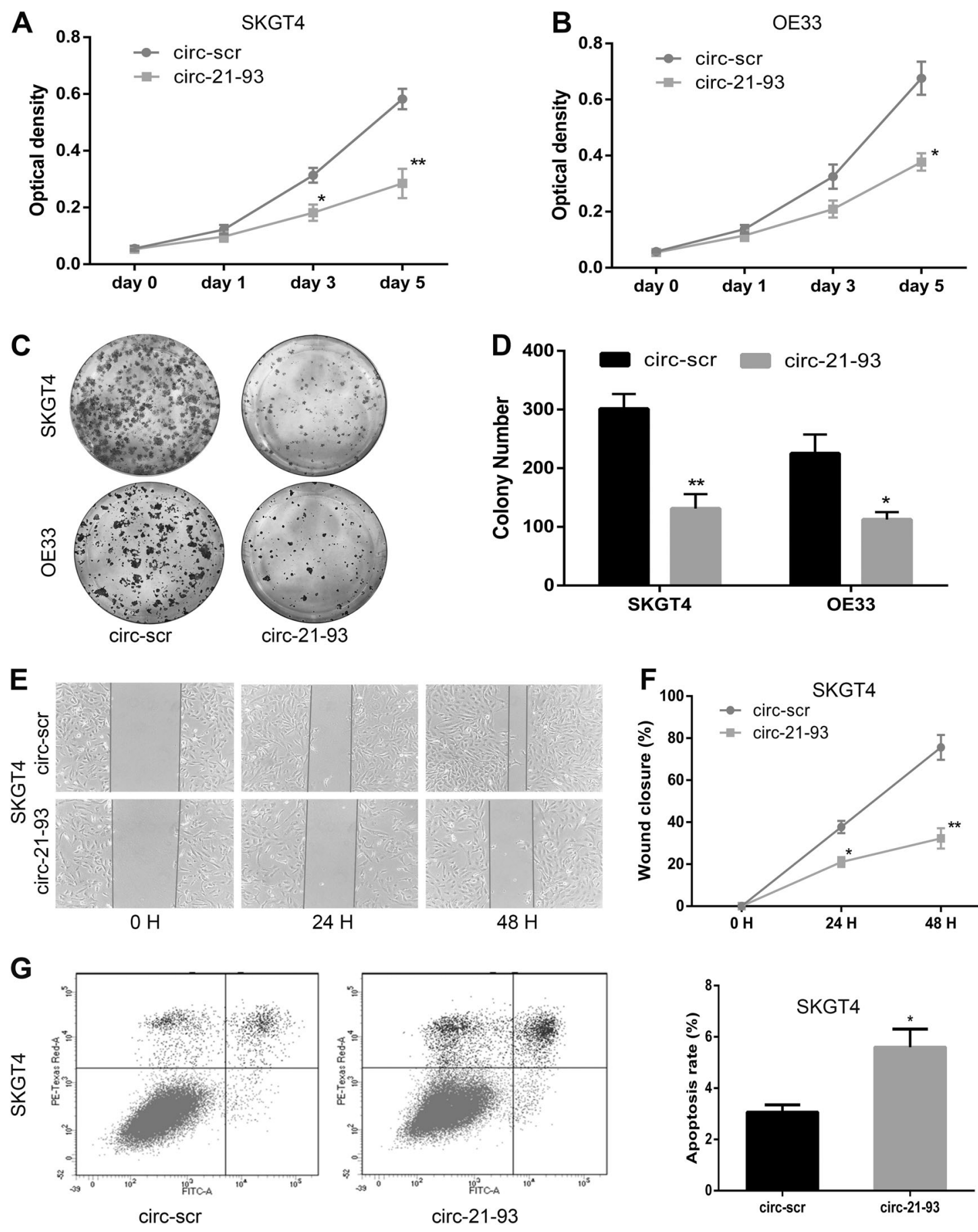

Fig. 5 Synthetic circular multi-miR sponge inhibits proliferation and migration but promotes apoptosis of EC cells in vitro. A, B Results of WST-1 assay for proliferation after transfection with either circ-scr or circ-21-93. Inhibition of proliferation by binding of endogenous miR21 and miR-93 in SKGT4 cell lines at day 3 and day $5(P=0.0272$ and 0.0093 , respectively). In OE33 cells, circ-21-93 significantly inhibited proliferation at day 5 relative to circ-scr $(P=0.0109)$. C, D Representative images and quantification results of colony formation. Cells transfected with circ-21-93 showed lower colony
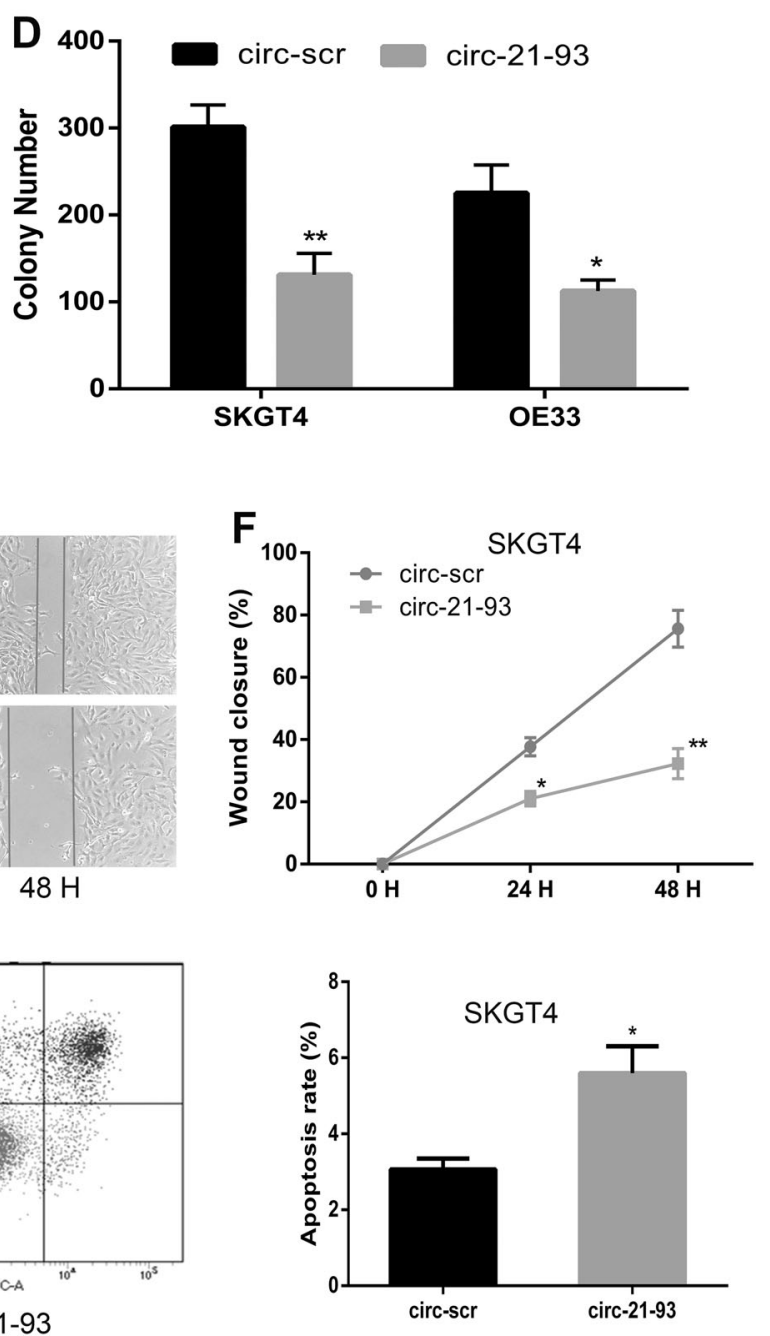

numbers in both SKGT4 and OE33 cell lines. E Representative images of scratch assays in SKGT4 cells transfected with either circ-scr or circ-21-93. F Line chart depicting scratch healing rates of SKGT4 cells after transfection of circ-21-93. At 24 and $48 \mathrm{~h}$, SKGT4 wound closure in the circ-21-93 group was significantly lower than in the circ-scr group $(P=0.0306$ and 0.0086 , respectively). G Representative images and quantification results of flow cytometry to evaluate apoptosis induction by circ-21-93 in SKGT4 cells. $* p<0.05 ; * *<0.01$ 

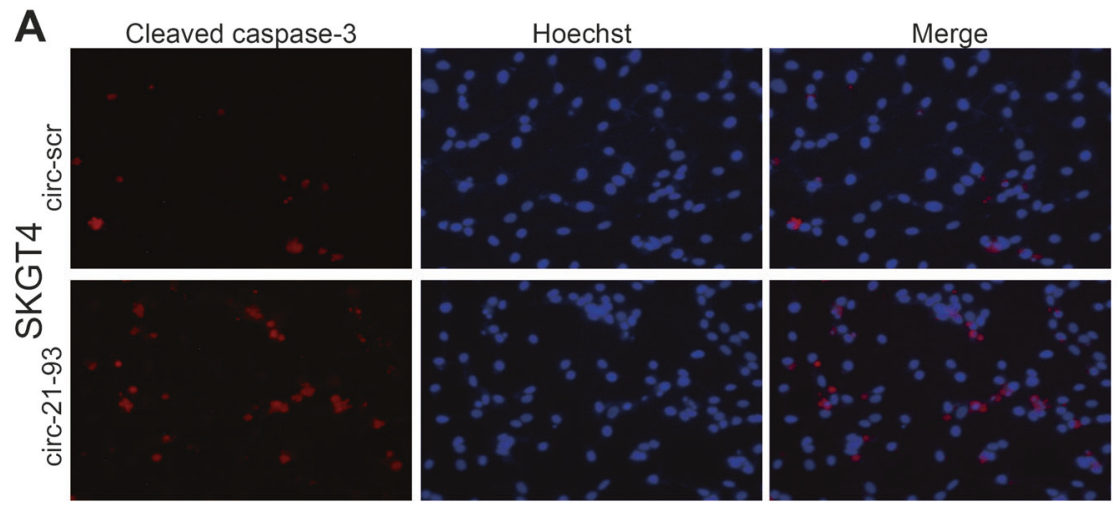

B
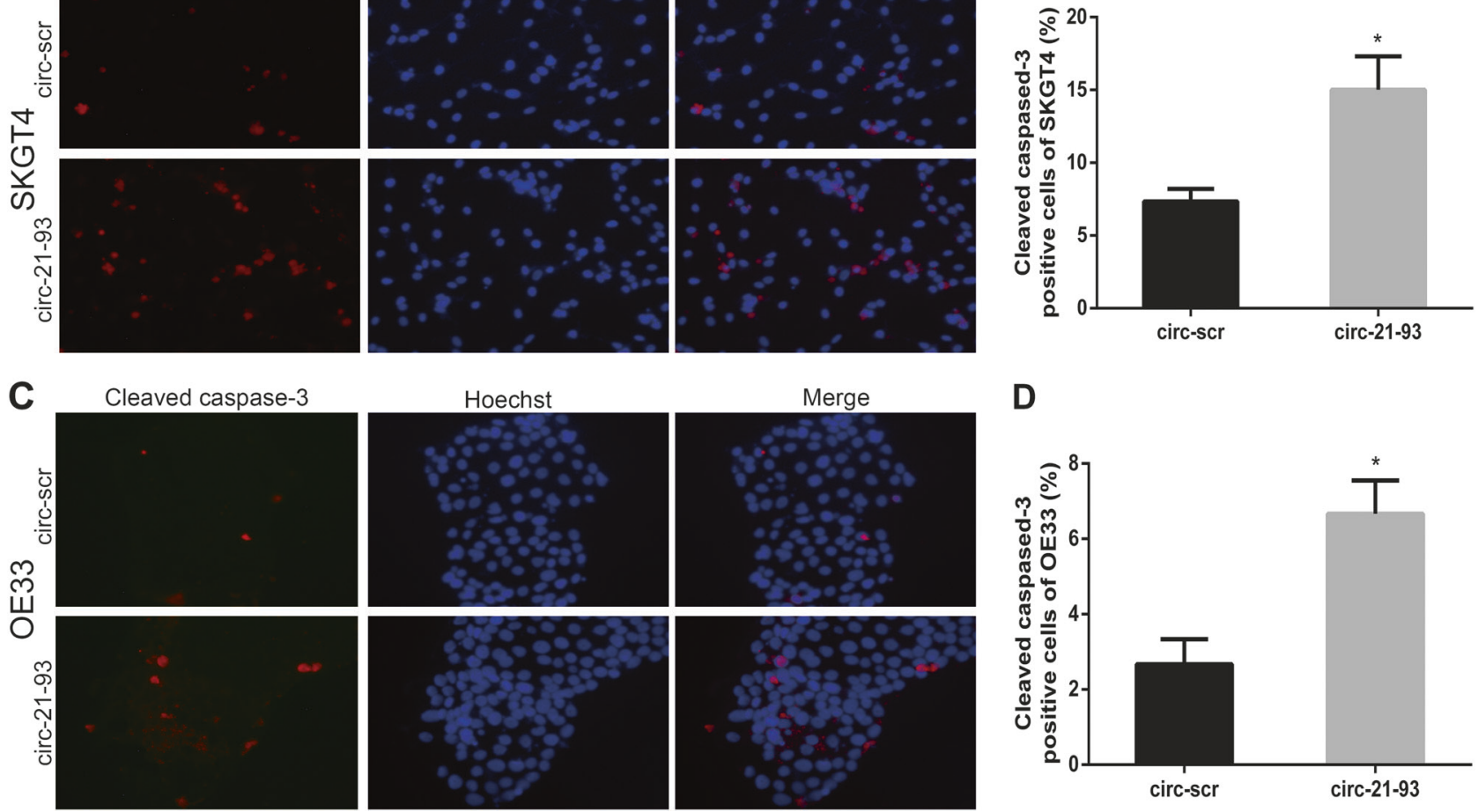

D

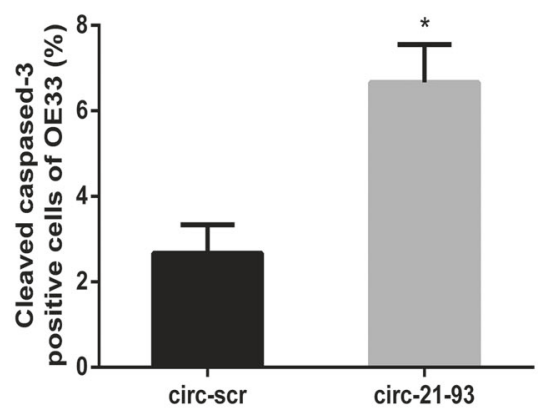

Fig. 6 Immunofluorescence staining of cleaved caspase-3 in EC cells. A, B Representative images and quantification results of SKGT4 cells transfected with circ-scr or circ-21-93. Relative to circ-scr, circ-21-93 induced significantly higher levels of cleaved caspase-3 $(P=0.0362)$.

C, D Representative images and quantification results of OE33 cells treated with either circ-scr or circ-21-93. Cleaved caspase-3 positive cells comprise a significantly higher proportion in circ-21-93- $v s$. circscr-treated cells $(P=0.0224)$. $* p<0.05$
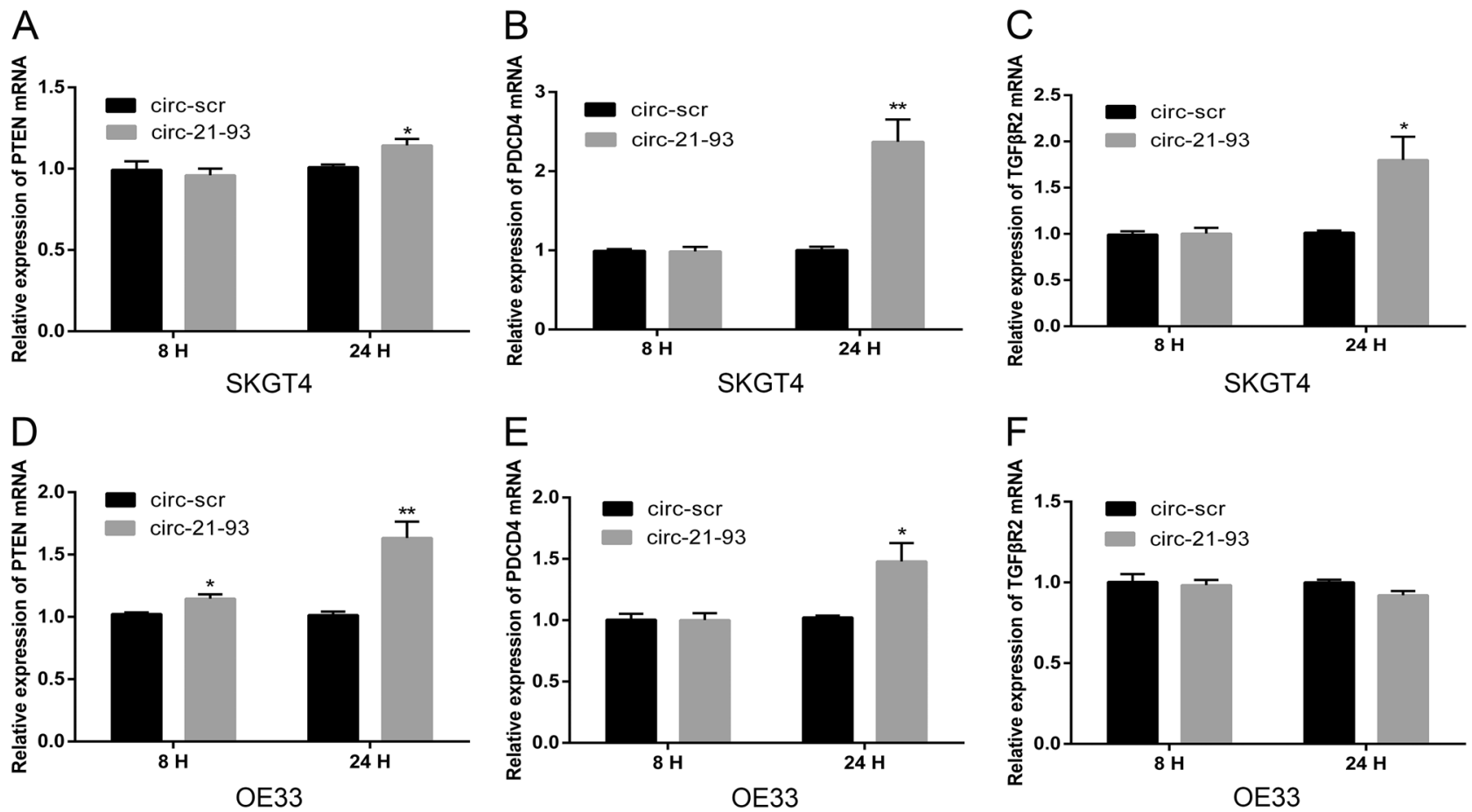

Fig. 7 Expression levels of tumor suppressor genes targeted by miR21 and miR-93. A-C In SKGT4 cells, mRNA expression levels of PTEN, PDCD4 and TGF $\beta R 2$ in circ-21-93 vs. circ-scr at 8 and $24 \mathrm{~h}$.

D-F After transfecting circ-21-93 or circ-scr into OE33 cells, mRNA levels of PTEN, PDCD4 and TGF $\beta$ R2 were measured by qRT-PCR at 8 and 24 h, respectively. $* p<0.05 ; * * p<0.01$ 
increased due to sequestration of miRs by our circular multi-miR sponge. Therefore, we measured expression levels of these tumor suppressor genes after transfection with circ-21-93 or a scrambled control (circ-scr) in SKGT4 and OE33 cells. Results showed that at $8 \mathrm{~h}$ after transfection, other than a slight change in PTEN expression induced by circ-21-93 in OE33 cells, these target mRNA expression levels were not altered. However, at $24 \mathrm{~h}$ after circ-21-93 transfection, PTEN, PDCD4 and TGFßR2 mRNA expression were significantly upregulated relative to circ-scr in SKGT4 cells (Fig. 7), establishing that circ-21-93 exerts functional activity against miR-21 and miR-93 via their downstream target tumor suppressor genes.

\section{The synthetic circular multi-miR sponge suppresses xenografted tumor growth in vivo}

To establish the therapeutic potential of synthetic our circular multi-miR sponge in vivo, we injected SKGT4 cells containing circ-21-93 or circ-scr subcutaneously into the dorsal flanks of nude mice and allowed to proliferate for four weeks. Tumor size was measured three times per week after tumor formation. Figure $8 \mathrm{~A}$ is a representative image of xenografted tumors in nude mice on day 33 after injection. Left-sided xenografted dorsal flank tumors arose from SKGT4 cells transfected with circ-21-93; right-sided tumors arose from SKGT4 cells with transfected with circ-scr. Relative to the circ-scr group, we found that tumors derived from SKGT4 cells contraining circ-21-93 grew significantly more slowly (Fig. 8B).

\section{Discussion}

We engineered and successfully constructed a synthetic circular multi-miR sponge directed against miR-21 and miR-93. The mechanistic effectiveness of this sponge was validated by luciferase assays, which proved that circ-21-93 effectively sequestered endogenous miR-21 and miR-93 in a dose-dependent manner. Moreover, we showed that our multi-miR sponge exerted tumor-suppressive effects on cellular functions including proliferation, migration, and apoptosis. To our knowledge, this is the first report of any synthetic multi-miR circular sponge used in any type of cancer.

In recent decades, accumulating evidence has shown that miRs inhibit target mRNA expression post-transcriptionally, either by repressing translation or causing mRNA degradation; moreover, miRs have been shown to impact the development and progression of almost all tumor types [24]. It is also known that more than one miR may initiate and drives tumor progression, and that some miRs function synergistically $[25,26]$. Therefore, in achieving
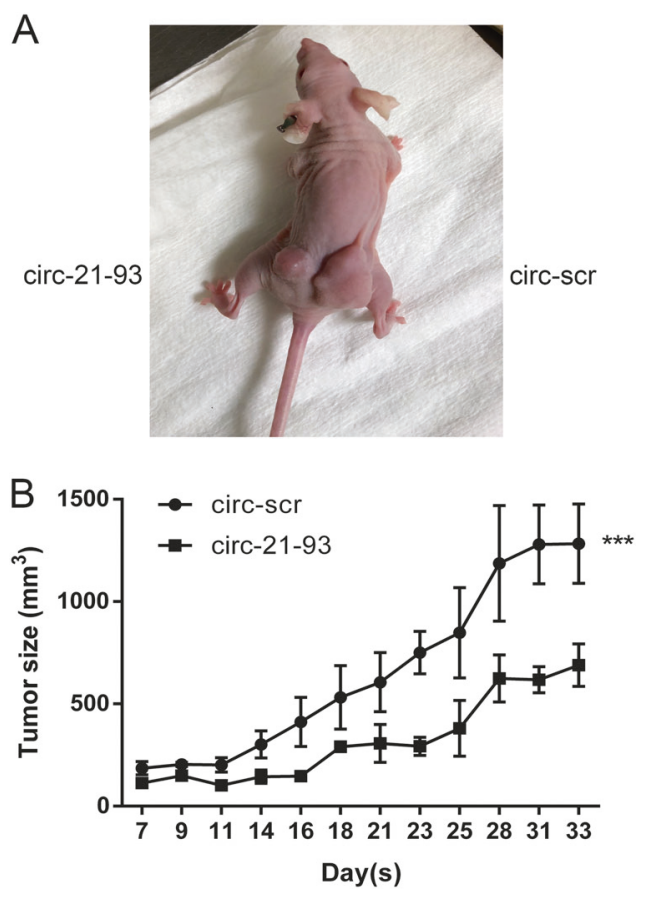

Fig. 8 Synthetic circular multi-miR sponge inhibits tumorigenesis in vivo. A Representative image of xenografted tumor formation on day 33 after inoculation. Left: injected with SKGT4 cells with circ-2193. Right: injected with SKGT4 cells transfected with circ-scr. B Growth curves of xenografted tumors derived from SKGT4 cells transfected with either circ-21-93 or circ-scr ( $n=4$ for each group). $* * * p<0.001$

miR loss-of-function, it would be expected that simultaneous inhibition of multiple miRs would be more effective than inhibition of a single miR. In the current study, we focused on miR-21 and miR-93, two established onco-miRs in EC. MiR-21, one of first known oncogenic miRs, is overexpressed in multiple tumors and regulates with pathways by binding to tumor suppression genes such as PDCD4, Bcl-2, and is involved in several important cellular processes [27-29]. In EC, knockdown of miR-21 significantly increased PTEN expression, reduced cell proliferation, invasion and migration [17]. In addition, overexpression of miR-21 was related to neoplastic transformation and progression in esophageal adenocarcinoma [30]. Similarly, previous researchers (including us) reported that miR-93 was overexpressed in EC cells and exerted cancer-promoting effects in vitro and in vivo [18, 31]. Moreover, miR-93 promoted radiation resistance by binding to the 3'-UTR sequence of B-cell translocation gene 3 (BTG3) mRNA [32]. Therefore, these two miRs were selected as representative esophageal onco-miRs against which to synthesize a multi-miR sponge. Consistent with previous studies, we also found that miR-21 and miR-93 were overexpressed in esophageal cancer cells.

MiR sponges are natural or synthetic RNAs that competitively inhibit miRs via multiple or tandem complementary 
sites [4]. Recently, growing evidence has shown that endogenous circRNAs can function as natural miR sponges, regulating target mRNA expression by absorbing miRs. One such circRNA, ciRs-7, contains 73 conserved single miR-7 binding sites, thereby blocking miR-7-induced tumor suppression by antagonizing the binding of miR-7 to its downstream oncogenes, EGFR and RAF1 [33]. Similarly, overexpression of circPVT1 effectively promoted gastric cancer cell proliferation by acting as a sponge for members of the miR-125 family [34]. These novel findings indicate that the function of circRNAs as miR sponges represents a widespread phenomenon. More importantly, the specific structures and functions of naturally occurring circRNAs provided us with the inspiration to design and construct artificial circular miR sponges to specifically inhibit known onco-miRs in cancer cells. In our previous study, we synthesized a circular RNA containing only a single miR binding site, for miR-21, and tested it in gastric cancer cells. That circular RNA effectively competed away native miR-21 activity on multiple downstream targets, including DAXX, while also inhibiting cancer cell proliferation [35]. In the current study, we included complementary binding sites for multiple miRs, specifically miR-21 and miR-93. We found that this synthetic circular multi-miR sponge competed successfully against both miR-21 and miR-93 simultaneously. At the functional level, our multi-miR sponge inhibited cell proliferation, migration, and tumor growth while promoting apoptosis, consistent with the induction of a less malignant phenotype. In addition, these effects were shown to be dosedependent. Moreover, our circular RNA sponge exerted more stable and durable effects than its linear RNA sponge counterpart, while specifically inhibiting the expression of miR-21 and miR-93 downstream target mRNAs. Taken together, these results prove that it is feasible to engineer and artificially construct synthetic circRNAs to function as miR sponges against cancers.

The specific protocol for constructing stable circular miRs sponges also represents an important and critical discovery of our study. RNA circularization involves intramolecular formation of 3', 5'-phosphodiester bonds between the 3' acceptor- and 5' donor-terminus of the linear RNA molecule. For methods of RNA circularization, chemical and enzymatic ligation strategies have been used before [36, 37]. Based on our previous study [35], enzymatic ligation was employed to combine single-stranded linear RNA oligonucleotides in the current study. After dephosphorylation by calf intestinal phosphatase and phosphorylation by T4 polynucleotide kinase, T4 RNA ligase 1 was chosen to assist in the circularization of singlestranded RNA molecules, a method that was originally introduced in the early 1970s [38]. RT-PCR and Sanger sequencing confirmed that the circular multi-miR sponge was successfully and correctly constructed. Notably, RNase
$\mathrm{R}$ proved that the circular RNA was resistant to miRmediated RNA destabilization and more stable than its linear counterpart, making it potentially more effective than linear miR sponges, which have been studied before. These results indicate that the method we used to synthetize circular RNA is feasible and practical. However, our current method is more suitable to small and medium-sized RNAs, less than $500 \mathrm{nt}$, resulting in limited numbers of miR binding sites. Longer stable circular miR sponges deserve further exploration in future studies.

In conclusion, we have developed and synthesized a novel artificial circular multi-miR sponge which achieves loss-of-function of miR-21 and miR-93. Our research proves that it is convenient and feasible to construct synthetic circular RNA sponges as tools to counteract miR functions. Although this technology is not yet fully mature and still has imperfections, such as a limited number of miR binding sites, varied yield of ligation for RNA circularization, and issues of potential toxicity, this discovery provides a new direction for future miR-directed molecular therapy and is worthy of continued investigation.

Funding: SJM was supported by NIH grants CA190040, CA211457, DK118250, and the Emerson Research Foundation; Dr. Meltzer is the Harry and Betty Myerberg Professor of Gastroenterology and an American Cancer Society Clinical Research Professor.

\section{Compliance with ethical standards}

Conflict of interest The authors declare that they have no conflict of interest.

Publisher's note: Springer Nature remains neutral with regard to jurisdictional claims in published maps and institutional affiliations.

\section{References}

1. Bartel DP. MicroRNAs: target recognition and regulatory functions. Cell. 2009;136:215-33.

2. Farazi TA, Spitzer JI, Morozov P, Tuschl T. miRNAs in human cancer. J Pathol. 2011;223:102-15.

3. Bak RO, Mikkelsen JG. miRNA sponges: soaking up miRNAs for regulation of gene expression. Wiley Interdiscip Rev RNA. 2014;5:317-33.

4. Ebert MS, Neilson JR, Sharp PA. MicroRNA sponges: competitive inhibitors of small RNAs in mammalian cells. Nat Methods. 2007:4:721-6.

5. Hansen TB, Jensen TI, Clausen BH, Bramsen JB, Finsen B, Damgaard CK, et al. Natural RNA circles function as efficient microRNA sponges. Nature. 2013;495:384-8.

6. Jeck WR, Sharpless NE. Detecting and characterizing circular RNAs. Nat Biotechnol. 2014;32:453-61.

7. Memczak S, Jens M, Elefsinioti A, Torti F, Krueger J, Rybak A, et al. Circular RNAs are a large class of animal RNAs with regulatory potency. Nature. 2013;495:333-8.

8. Han D, Li J, Wang H, Su X, Hou J, Gu Y, et al. Circular RNA circMTO1 acts as the sponge of microRNA-9 to suppress 
hepatocellular carcinoma progression. Hepatology. 2017;66: $1151-64$.

9. Ibraheem D, Elaissari A, Fessi H. Gene therapy and DNA delivery systems. Int J Pharm. 2014;459:70-83.

10. Kotterman MA, Chalberg TW, Schaffer DV. Viral vectors for gene therapy: translational and clinical outlook. Annu Rev Biomed Eng. 2015;17:63-89.

11. Bray F, Ferlay J, Soerjomataram I, Siegel RL, Torre LA, Jemal A. Global cancer statistics 2018: GLOBOCAN estimates of incidence and mortality worldwide for 36 cancers in 185 countries. CA Cancer J Clin. 2018;68:394-424.

12. Khan AA, Advani J, Patel K, Nanjappa V, Datta KK, Solanki $\mathrm{HS}$, et al. Chronic exposure to cigarette smoke and chewing tobacco alters expression of micrornas in esophageal epithelial cells. Microrna. 2018;7:28-37.

13. Dong J, Buas MF, Gharahkhani P, Kendall BJ, Onstad L, Zhao $\mathrm{S}$, et al. Determining risk of barrett's esophagus and esophageal adenocarcinoma based on epidemiologic factors and genetic variants. Gastroenterology. 2018;154:1273-81.

14. Hemmatzadeh M, Mohammadi H, Karimi M, Musavishenas MH, Baradaran B. Differential role of microRNAs in the pathogenesis and treatment of Esophageal cancer. Biomed Pharmacother. 2016;82:509-19.

15. Mathe EA, Nguyen GH, Bowman ED, Zhao Y, Budhu A, Schetter AJ, et al. MicroRNA expression in squamous cell carcinoma and adenocarcinoma of the esophagus: associations with survival. Clin Cancer Res. 2009;15:6192-200.

16. Lu Z, Liu M, Stribinskis V, Klinge CM, Ramos KS, Colburn $\mathrm{NH}$, et al. MicroRNA-21 promotes cell transformation by targeting the programmed cell death 4 gene. Oncogene. 2008;27:4373-9.

17. Li P, Mao WM, Zheng ZG, Dong ZM, Zheng ZG, Ling ZQ. Down-regulation of PTEN expression modulated by dysregulated miR-21 contributes to the progression of esophageal cancer. Dig Dis Sci. 2013;58:3483-93.

18. Kan T, Sato F, Ito T, Matsumura N, David S, Cheng Y, et al. The miR-106b-25 polycistron, activated by genomic amplification, functions as an oncogene by suppressing p21 and Bim. Gastroenterology. 2009;136:1689-700.

19. Fu X, He Y, Wang X, Peng D, Chen X, Li X, et al. Overexpression of miR-21 in stem cells improves ovarian structure and function in rats with chemotherapy-induced ovarian damage by targeting PDCD4 and PTEN to inhibit granulosa cell apoptosis. Stem Cell Res Ther. 2017;8:187.

20. Yang W, Bai J, Liu D, Wang S, Zhao N, Che R, et al. MiR-93-5p up-regulation is involved in non-small cell lung cancer cells proliferation and migration and poor prognosis. Gene. 2018;647:13-20.

21. Liang H, Wang F, Chu D, Zhang W, Liao Z, Fu Z, et al. miR-93 functions as an oncomiR for the downregulation of PDCD4 in gastric carcinoma. Sci Rep. 2016;6:23772.

22. Lyu X, Fang W, Cai L, Zheng H, Ye Y, Zhang L, et al. TGFßR2 is a major target of miR-93 in nasopharyngeal carcinoma aggressiveness. Mol Cancer. 2014;13:51.
23. Yu Y, Kanwar SS, Patel BB, Oh PS, Nautiyal J, Sarkar FH, et al. MicroRNA-21 induces stemness by downregulating transforming growth factor beta receptor 2 (TGFbetaR2) in colon cancer cells. Carcinogenesis. 2012;33:68-76.

24. Ambros V. The functions of animal microRNAs. Nature. 2004;431:350-5.

25. Kluiver J, Slezak-Prochazka I, Smigielska-Czepiel K, Halsema N, Kroesen BJ, Berg A. Generation of miRNA sponge constructs. Methods. 2012;58:113-7.

26. Kent OA, Mendell JT. A small piece in the cancer puzzle: microRNAs as tumor suppressors and oncogenes. Oncogene. 2006;25:6188-96.

27. Motoyama K, Inoue H, Mimori K, Tanaka F, Kojima K, Uetake $\mathrm{H}$, et al. Clinicopathological and prognostic significance of PDCD4 and microRNA-21 in human gastric cancer. Int J Oncol. 2010;36:1089-95.

28. Si ML, Zhu S, Wu H, Lu Z, Wu F, Mo YY. miR-21-mediated tumor growth. Oncogene. 2007;26:2799-803.

29. Yan LX, Wu QN, Zhang Y, Li YY, Liao DZ, Hou JH, et al. Knockdown of miR-21 in human breast cancer cell lines inhibits proliferation, in vitro migration and in vivo tumor growth. Breast Cancer Res. 2011;13:R2.

30. Hezova R, Kovarikova A, Srovnal J, Zemanova M, Harustiak T, Ehrmann J, et al. Diagnostic and prognostic potential of miR-21, miR-29c, miR-148 and miR-203 in adenocarcinoma and squamous cell carcinoma of esophagus. Diagn Pathol. 2015;10:42.

31. Ansari $\mathrm{MH}$, Irani $\mathrm{S}$, Edalat $\mathrm{H}$, Amin $\mathrm{R}$, Roushandeh $\mathrm{AM}$. Deregulation of miR-93 and miR-143 in human esophageal cancer. Tumour Biol. 2016;37:3097-103.

32. Cui H, Zhang S, Zhou H, Guo L. Direct downregulation of b-cell translocation gene 3 by microRNA- 93 is required for desensitizing esophageal cancer to radiotherapy. Dig Dis Sci. 2017;62:1995-2003.

33. Weng W, Wei Q, Toden S, Yoshida K, Nagasaka T, Fujiwara $\mathrm{T}$, et al. Circular RNA ciRS-7-A promising prognostic biomarker and a potential therapeutic target in colorectal cancer. Clin Cancer Res. 2017;23:3918-28.

34. Chen J, Li Y, Zheng Q, Bao C, He J, Chen B, et al. Circular RNA profile identifies circPVT1 as a proliferative factor and prognostic marker in gastric cancer. Cancer Lett. 2017;388:208-19.

35. Liu X, Abraham JM, Cheng Y, Wang Z, Wang Z, Zhang G, et al. Synthetic circular RNA functions as a miR-21 sponge to suppress gastric carcinoma cell proliferation. Mol Ther Nucleic Acids. 2018;13:312-21.

36. Dolinnaya NG, Blumenfeld M, Merenkova IN, Oretskaya TS, Krynetskaya NF, Ivanovskaya MG, et al. Oligonucleotide circularization by template-directed chemical ligation. Nucleic Acids Res. 1993;21:5403-7.

37. Rezaian MA. Synthesis of infectious viroids and other circular RNAs. Current Issues Molec Biol. 1999;1:13-20.

38. Silber R, Malathi VG, Hurwitz J. Purification and properties of bacteriophage T4-induced RNA ligase. Proc Natl Acad Sci USA. 1972;69:3009-10. 